\title{
Surveillance of sexually transmitted diseases in France: recent trends and incidence
}

\author{
Laurence Meyer, Véronique Goulet, Véronique Massari, Agnes Lepoutre-Toulemon
}

\begin{abstract}
Objective-To present recent trends in sexually transmitted diseases (STDs) in France and to estimate the gonorrhoea incidence in 1990.

Methods-Trends were analysed from data of three surveillance systems: (1) STD clinics: number of diagnoses, (2) a sentinel voluntary General Practitioner (GP) network: mean number of acute male urethritis/week/GP, characteristics of the notified urethritis (age, presence of discharge, sexual orientation), (3) a sentinel voluntary laboratory network: mean number of gonorrhoea isolates/ month/laboratory, characteristics of patients with positive isolates (sex, age, site of sampling) and of strains (PPNG and TRNG rates). To estimate the gonorrhoea incidence in France in 1990, results of a study held among a national sample of laboratories were used, combined with data from surveillance systems and specific studies.
\end{abstract}

Results-Decreasing trends in gonorrhoea in STD clinics and in the laboratory network as well as in acute male urethritis in the GP network have been observed since implementation of the networks in 1985. The rate of PPNG strains has regularly increased in the laboratory network to reach $14 \%$ in 1991. Data suggest that the incidence in some acute non recurrent STDs could have increased among homo/bisexual men since 1988. Chlamydia trachomatis is now the most frequent diagnosis in STD clinics. Estimation of male gonorrhoea incidence rate in France in 1990 of $74 / 100000$ inhabitants (15-59 years) is consistent with figures observed in England and Wales, where the age distribution is very similar. On the other hand, the estimated female gonorrhoea incidence rate of $14 / 100000$, which concerns only microbiologically ascertained cases, is one third in France than that observed in England and Wales.

Conclusion-The consistency of the decreasing trends in gonorrhoea and acute male urethritis observed from the different networks reduces the possibility of a bias due to any change in notification or in prescription. Trends in Chlamydia trachomatis will be better appraised in the near future with the recent implementation of new systems. The French STD surveillance appears quite satisfactory for male infections and has been able to show a marked decrease in the incidence of some STDs in the last years. Surveillance of female STDs is to be improved, in terms of monitored diagnoses and selected health care facilities. Differences between the female gonorrhoea incidence rate observed in England and Wales and the one computed for France could be attributed to differences in contact tracing policies between the two countries or to differences in sexual lifestyles.

(Genitourin Med 1994;70:15-21)

\section{Introduction}

Surveillance of sexually transmitted diseases (STDs) is of particular importance in France, since it is the European country with the greatest number of AIDS cases (25 555 registered cases on June 1993, that is 465 cases per million inhabitants). ${ }^{1}$

A French pecularity is the need for multiple data sources, as a variety of medical specialists (General Practitioners (GPs), gynaecologists, dermatologists) and clinical sites, both public and private, are involved in STD care. A compulsory system of notification for cases of syphilis, gonorrhoea, chancroid and lymphogranuloma venereum (LGV) dates from a law passed in 1942. Its purpose was primarily the control of infection rather than epidemiological surveillance. It was shown in $1978^{2}$ that although around $80 \%$ of cases of gonorrhoea and syphilis were diagnosed by private practitioners (GPs and others), more than $90 \%$ of notifications came from STD clinics. This underestimation of cases from compulsory notification has also been reported in some other European countries. ${ }^{3}$ Since then no attempt has been made in France to enhance the notifications. Instead, at the beginning of the eighties, several surveillance systems were implemented in samples of different types of health care services. Voluntary GP and laboratory networks were set up with the aim to monitor national trends in male acute urethritis and laboratory diagnosed gonorrhoea cases. Data from all the STD clinics have also been collected annually by the Ministry of Health since 1985 , mainly in order to assess clinical activity. Some modifications in the surveillance have been introduced after an evaluation in 1989.4

Several sources of data are now available, \\ 29 September 1993
}


with the benefit of hindsight, to allow a synthesis of the information, both in terms of trends and incidence. The objectives of this paper are to synthetise the recent trends in STDs in France, excluding HIV infections, and to estimate the gonorrhoea incidence in the year 1990 .

\section{Methods}

\section{A-Analysis of trends}

The following sources have been used in order to look for temporal trends in the incidence of STDs and in the M/F (male/female) sex-ratio and age of the cases. Reference will also be made to a number of ad hoc studies, mostly performed within each system.

(1) Compulsory notification Although the compulsory notification system dates back to 1942, we did not attempt to identify national trends in incidence owing to the incompleteness and inconsistency of reporting among private practitioners. Only data notified by Paris STD clinics since 1971 could easily be abstracted from it and are presented here, as completeness in STD clinics has been previously found to be satisfactory. ${ }^{2}$

(2) STD clinics $^{5}$ In France STD clinics are mandated by the 1942 law to provide free and anonymous diagnosis and treatment restricted to the four "classic" STDs (syphilis, gonorrhoea, chancroid and LGV). The yearly numbers of these diagnoses have been collected since 1985 by the Ministry of Health. As an increasing number of STD clinics now provide free care for some other STDs, numbers of cases of herpes simplex, genital warts and laboratory confirmed Chlamydia trachomatis have been reported since 1986.

In this paper, trends in numbers of cases of each diagnosis since 1985 are presented. The cases from Paris STD clinics since 1971 are presented separately. Sexual orientation (defined as homo, bi- or heterosexual) of cases in STD clinics is available only in Paris in 1990, from a specific survey which included all cases of a new suspected STD. ${ }^{6}$

(3) The French Communicable Diseases Network $(F C D N)^{7}$ This GP network was set up in 1984 under the auspices of the Ministry of Health and the National Institute of Health and Medical Research. The Sentinel General Practioners (SGPs), voluntary and unpaid, participate in the surveillance of eight communicable diseases or conditions, including acute male urethritis. They are asked to report at least once a week, on an online computer system (Minitel), the number of cases of each disease. In each case of urethritis, age and presence of a discharge are requested. ${ }^{8}$ Since April 1990, sexual orientation (defined as homo/bisexual or heterosexual) has been recorded. The functional size of the network was about 500 SGPs in 1991. National trends in incidence are estimated by trends in the average number of cases seen per SGP per week. As not all the SGPs communicate regularly at least once a week, two different corrections have to be made. The first one takes into account the number of days of participation for each GP. The second one corrects for differences between the regional distribution of SGPs having participated during a given week and the regional distribution of the French GP population. ${ }^{9}$

(4) The National Network of Gonorrhoea $(R E N A G O)^{10}$ This laboratory network was set up in 1985 for the surveillance of gonococcal infection and antibiotic resistant strains. It is made up of private and public laboratories, voluntary and unpaid, participating throughout the country. For each positive culture, age, sex, the anatomical site from which the specimen is obtained and the presence of symptoms are recorded by the participating laboratory. The number of genital specimens processed monthly, according to sex, is also reported. All the isolated strains are sent to the STD National Reference Center (Institut Alfred-Fournier, Paris) to assess $\beta$-lactamase production and the minimal inhibitory concentrations towards six antibiotics. The functional size of the network was about a hundred laboratories in 1991.

Trends in incidence are estimated by trends in the average number of gonorrhoea cases diagnosed per laboratory per month, taking into account the number of months of participation for each laboratory.

\section{$B$-Estimation of gonorrhoea incidence}

Several sources of data were successively used to estimate gonorrhoea incidence in $\mathbf{1 9 9 0 .}$

In order to assess the representativity of RENAGO, a study (the "National Laboratory Study") was performed in 1992 among a national random sample of one seventh of all the $\mathbf{4 1 9 3}$ microbiological laboratories. They were asked to state how many gonococcal infections they had diagnosed during 1991 . The response rate was $88 \%$. This study allowed us to compute a mean number of isolated gonorrhoea strains per laboratory per year. The sex-ratio observed in RENAGO was applied to these results to compute a mean number by sex. As no laboratory working with an STD clinic was included in the sample, this mean number was considered to reflect the "routine" activity of French laboratories. Cases from STD clinics had therefore to be added in order to estimate incidence by sex in microbiologically ascertained gonorrhoea in France.

In order to asses how many cases of gonorrhoea could be missed when only microbiologically ascertained cases are taken into account, data from other studies were used. One is a randomised community trial of prevention of STDs, ${ }^{11}$ where a sample of 263 GPs in six French departments (administrative area comprising about 600000 inhabitants) were asked to describe all cases of urethral or vaginal discharge among their patients. We will refer to this study as the "six Department-Study". The second one is a GP 


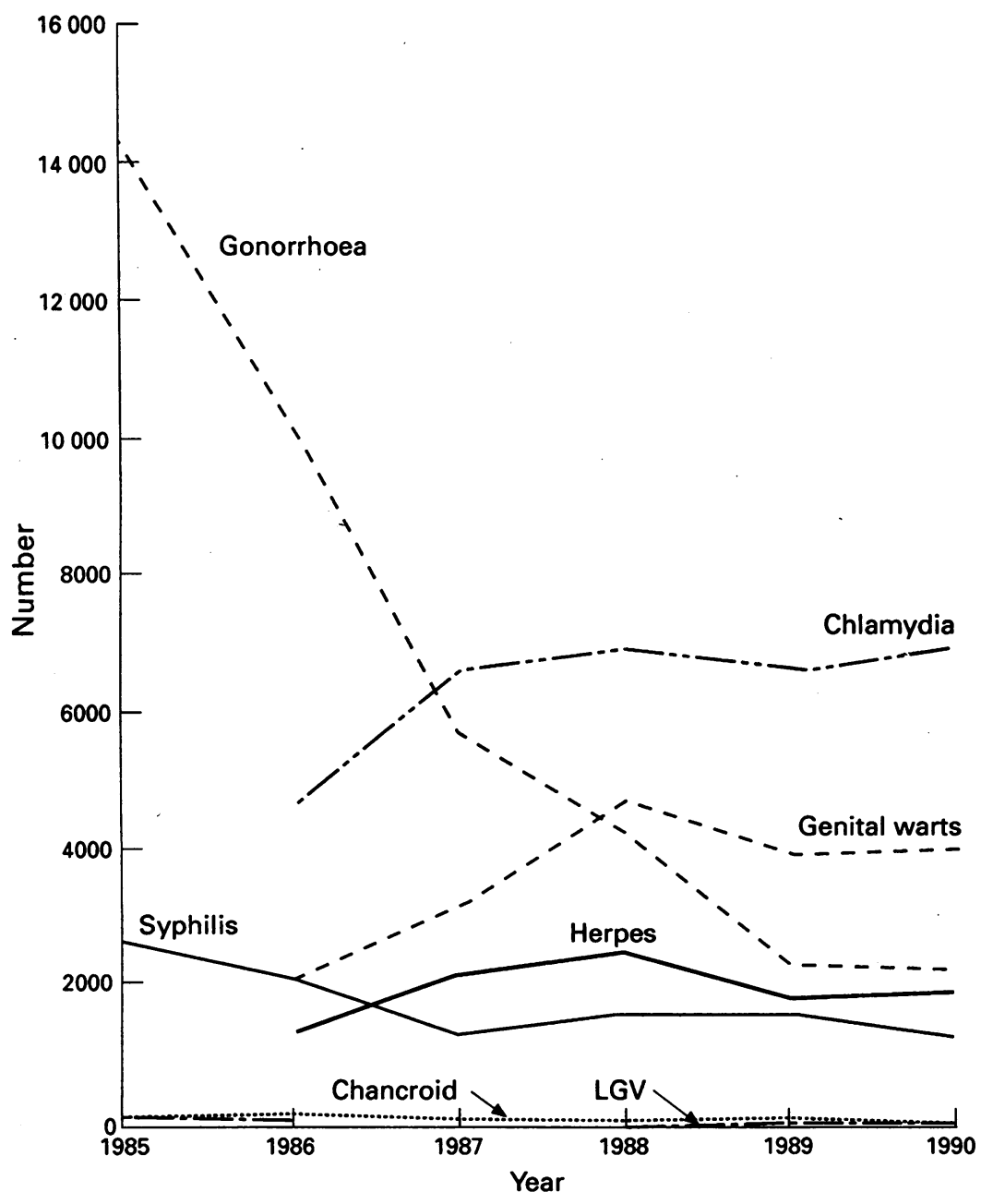

Figure 1 Trends in diagnosis in French STD clinics 1985-1990

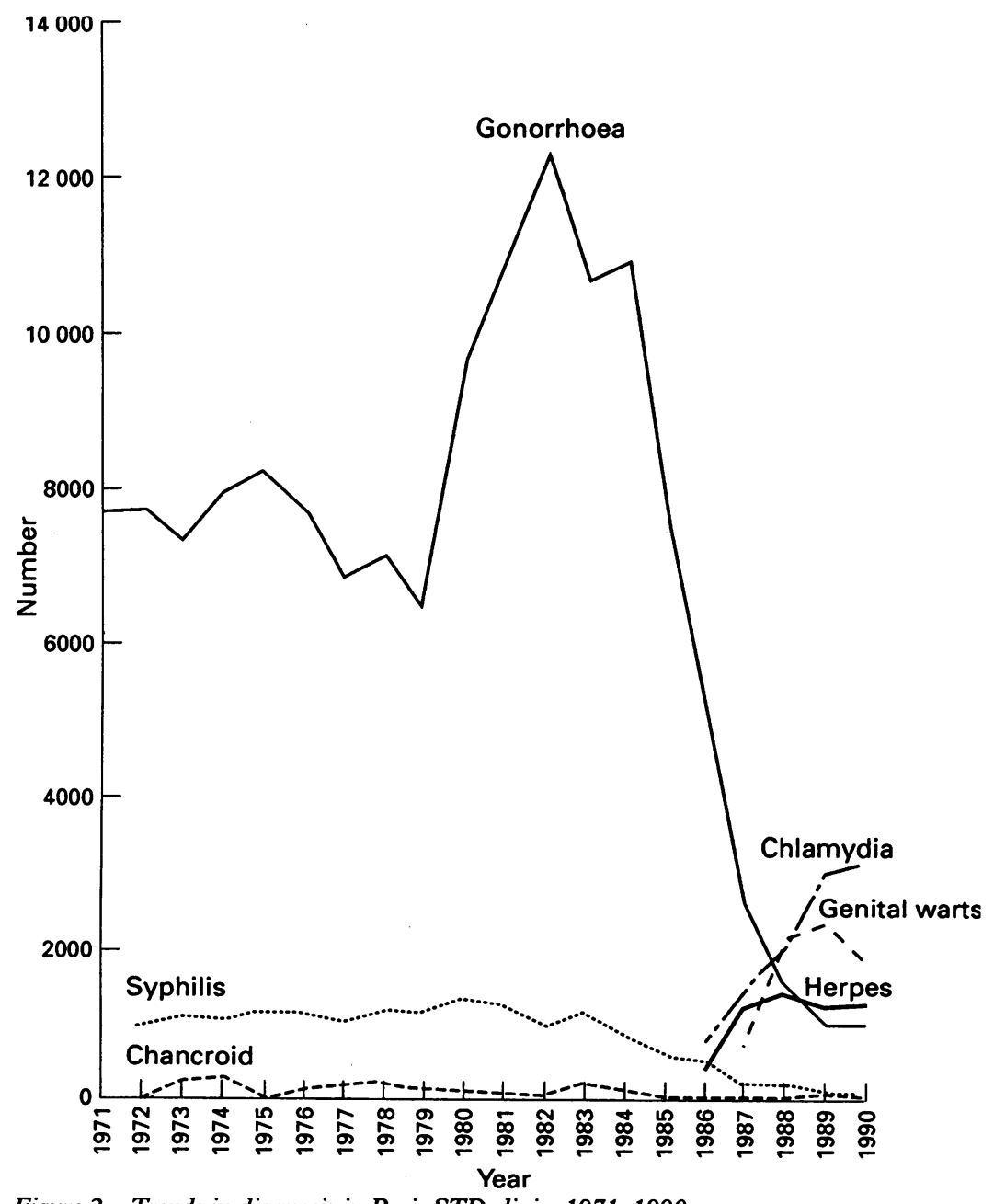

Figure 2 Trends in diagnosis in Paris STD clinics 1971-1990 network set up in Aquitaine, a French region (administrative area comprising about 3 millions of inhabitants), which has monitored male urethritis from October 1987 to October 1990.1213 Information about the diagnosis and its mode was collected from the hundred GPs who participated. We will refer to this study as the "Aquitaine network". These studies allowed us to estimate the proportion of male cases of gonorrhoea seen in general practice which are microbiologically diagnosed. Our estimation of male microbiologically ascertained gonorrhoea incidence was divided by this proportion to obtain the incidence in male gonorrhoea in France.

\section{Results}

(A) Recent trends in STDs

(1) STD diagnoses in STD clinics National data from STD clinics from 1985 to 1990 are shown on fig 1 . The total number of consultations has halved from 234000 in 1985 to around 121000 in 1989 and 1990. A sharp decrease in the number of cases of gonorrhoea has been observed since the outset. The proportion of cases of gonorrhoea among all the attenders has decreased from $6 \%$ in 1985 to $2 \%$ in 1990 . The data suggest a recent plateau since 1989 in both the number of attenders and gonorrhoea cases. The number of early syphilis cases decreased until 1987 and has fluctuated since then. The numbers of chancroid and LGV cases have been at a same low level (around a hundred cases per year) since 1985. The number of microbiologically diagnosed Chlamydia trachomatis cases has increased since 1986 , when recording began. The level seems to have stabilised. Chlamydia is now the most frequent diagnosis in STD clinics. It is probably still underestimated as not all the clinics offer testing to all their patients. No increase in the number of cases of herpes simplex and genital warts has been observed since 1988. Genital warts are now the second most frequently declared condition in French STD clinics.

In 1990, a male/female (M/F) sex-ratio of 2.0 was observed for gonorrhoea cases in STD clinics from 20 departments excluding Paris.

Data from the Paris STD clinics (fig 2) are available since 1971. A sharp increase in notification of cases of gonorrhoea was observed between 1979 and 1982. A fall in reported cases of gonorrhoea began in 1983 and continued until 1989. The data suggest a plateau in 1990. The global number of early syphilis cases in these clinics has declined since 1980: the number of secondary syphilis has regularly declined since 1980 , but data for primary syphilis show a slight increase in 1982-1983 followed by a continuous decrease afterwards. The number of chancroid infections has been low and stable since 1985. In Paris STD clinics, LGV cases are anecdotal, less than five cases per year. The number of microbiologically diagnosed Chlamydia trachomatis cases has regularly increased since 1986 when recording first began. Chlamydial infection is now the most 


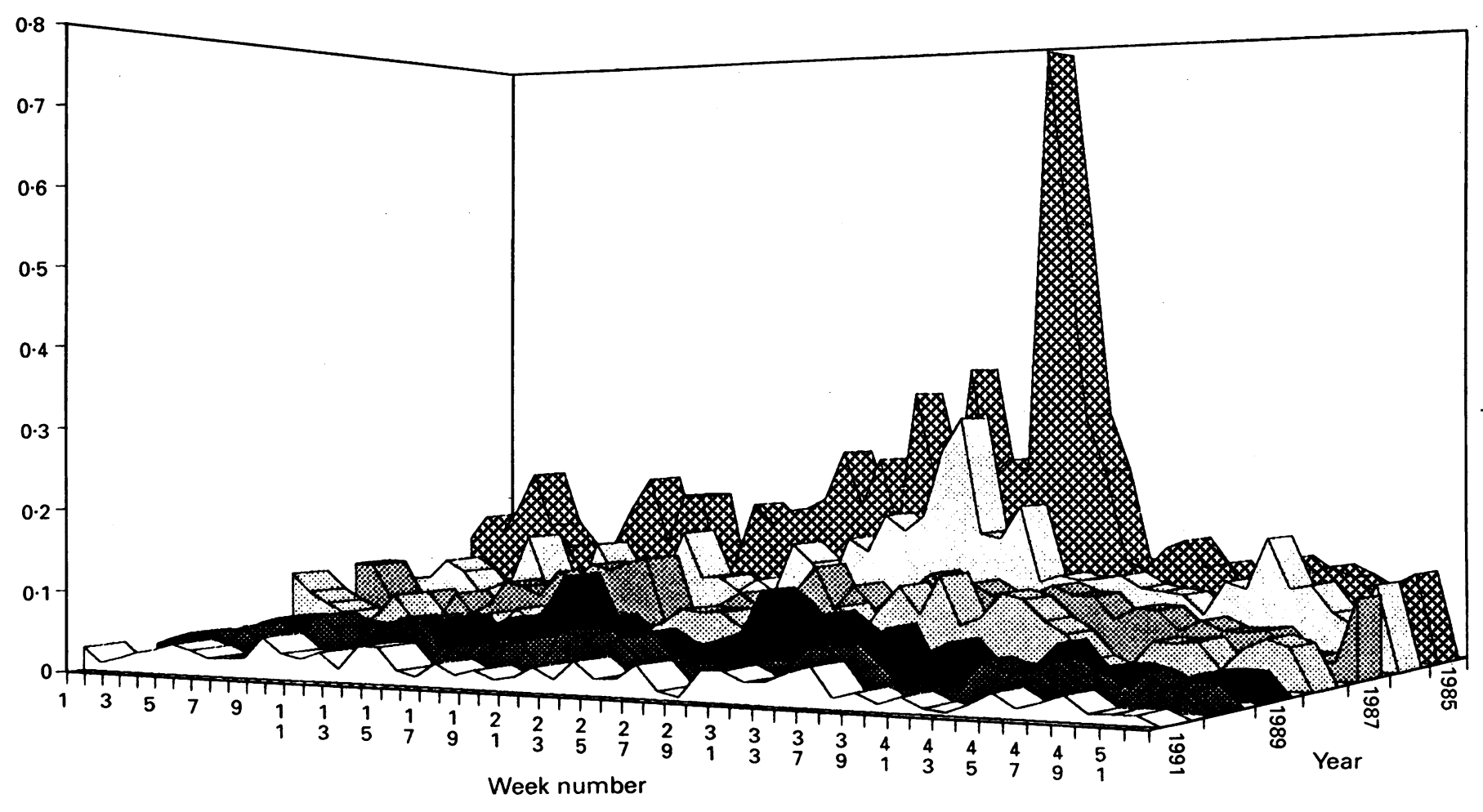

Figure 3 Trend in the mean number of urethritis cases per GP per week-the FCDN: 1985-1991

frequent diagnosis in Paris STD clinics. It is probably still underestimated by these figures, as not all the Paris clinics offer free chlamydia testing to all their patients.

Sex and age of gonorrhoea and syphilis cases diagnosed in Paris STD clinics are available only in 1986 and 1990 . In 1986 sexratio was $\mathbf{7 \cdot 2}$ for diagnosis of gonorrhoea and 7.5 for early syphilis. In 1990 it was respectively $9 \cdot 7$ and $6 \cdot 0$, higher for gonorrhoea than in 1986 and than in the non Parisian STD clinics in 1990. The age of male cases has significantly increased between 1986 and 1990 . Men with gonorrhoea or early syphilis were younger in 1986 (mean age of respectively 29.5 and 34.0 years) than in 1990 (respec- tively 31.2 and 42.0 years). In $1990,25 \%$ o male STD cases and $40 \%$ of male gonorrhoę cases were homo/bisexual. Homo/bisexuạ men with gonorrhoea were significantly younger (28.9 years) than heterosexual one (32.8 years). Men with rectal gonorrhoes were younger $(26.7$ years) than men with ure thral gonorrhoea ( $31 \cdot 6$ years).

No significant difference between 198 and 1990 in age of female cases could be noted. Mean age of women with gonorrhoea was $26 \cdot 7$ years in 1986 and $27 \cdot 7$ years in. 1990. Mean age of women with early syphilis was respectively 32.4 and 37.8 years.

In 1990 in Paris STD clinics, the rate of PPNG (Penicillinase Producing Neisseriog

Table 1 Trends in characteristics of male acute urethritis notified by Sentinel General Practitioners in the FCDN: 1985-1991

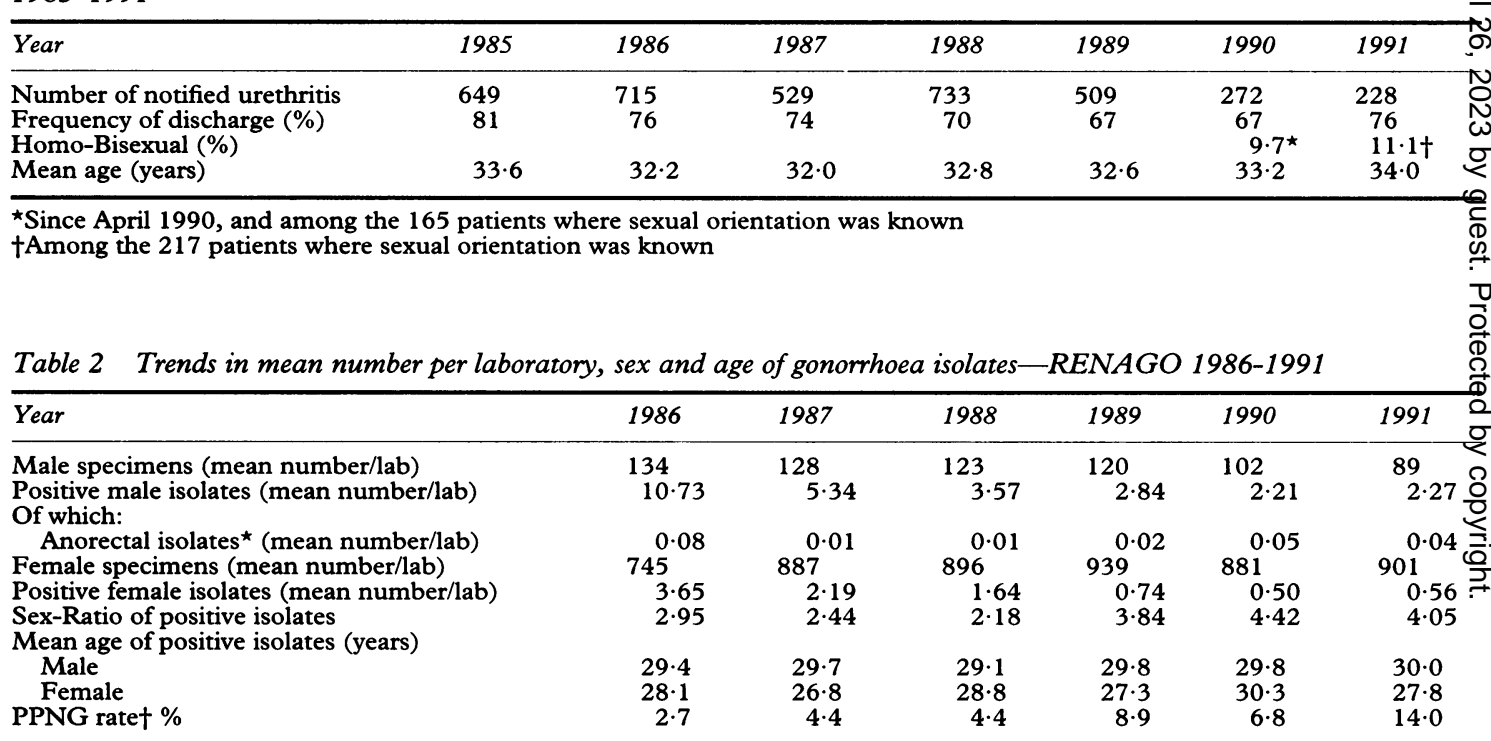

$\star$ Number of negative anorectal specimens not collected

†Penicillinase Producing Neisseria gonorrhoea 
gonorrhoea) strains varied between 10 and $30 \%$ according to the clinic.

(2) Acute male urethritis in the FCDN In this GP network, a continuing decline in the average number of cases of male urethritis seen weekly by SGP is observed from 1985 to 1991 (fig 3). The classic summer peak has disappeared. The proportion of cases of urethritis with a discharge (table 1) decreased regularly from $81 \%$ in 1985 to $67 \%$ in 1989 and 1990. In 1991 a significant increase to $76 \%$ was observed. In $1990,9 \cdot 7 \%$ of patients with urethritis were homo/bisexual, $11.1 \%$ in $1991(p=0.60)$. The corresponding figures for Paris area were $11.5 \%$ in 1990 and $16.0 \%$ in $1991(p=0.41)$, and for the rest of France $8.7 \%$ and $6.8 \%$. Mean age has changed with time, decreasing to 32 years in 1987 and increasing since then to 34 years in 1991 . Mean age did not differ in 1990-1991 between homo/bisexual and heterosexual men (respectively 33.4 and 33.9 years).

(3) Microbiologically diagnosed gonorrhoea cases in the RENAGO The RENAGO data (table 2) show a decline in the mean number of gonococcal strains isolated by participating laboratories from 1986 to 1990 , both in men and women. This decline cannot be attributed to a change in participating laboratories since the same pattern is observed when analysis is restricted to the laboratories which have consistently participated since the outset. The decrease in the number of genital tests performed among men does not fully explain the decrease observed in the number of positive male isolates. Figures in 1991 suggest a plateau.

The M/F sex-ratio decreased from 2.95 in 1986 to $2 \cdot 18$ in 1988 . This can be attributed to a more marked decrease in the rate of positive isolates among women than among men. Since then, it has increased and fluctuates now around 4. When restricting the 1990 data to the laboratories located in Paris area, sex-ratio was $6 \cdot 1$, lower than the one observed in Paris STD clinics.

RENAGO does not provide information on the sexual orientation of patients. However, the site of sampling for positive isolates is recorded. The mean number per laboratory in positive anorectal isolates has increased since 1988 from $0.01 /$ lab to 0.05 in 1990 and 0.04 in 1991 . The percentage of anorectal isolates among all male positive isolates has significantly increased since 1988 from $0.3 \%$ of the male cases to $2.6 \%$ in 1990 and $2 \cdot 1 \%$ in 1991 (p-trend for the last 4 years $=0.01)$. The same pattern of increase is observed when analysis is restricted to the laboratories which have consistently participated since 1986

There is no major trend in the mean age of men and women with positive isolates. Although they were very few, men with gonococcal proctitis in 1990 seemed older than men with gonococcal urethritis (mean age of respectively 37.0 and 29.6 years).

The rate of PPNG strains has dramatically increased from $2 \cdot 7 \%$ in 1986 to $14 \%$ in 1991 . Tetracycline resistant strains (TRNG) were first noticed in the network in $1989(2.3 \%$ of the strains) and persisted at the same level in 1990 and 1991.

(B) ESTIMATION OF GONORRHOEA INCIDENCE IN FRANCE IN 1990

In the National Laboratory Study, a mean number of $2 \cdot 27$ [1.87-2.67] gonorrhoea strains per laboratory was isolated by the respondent laboratories during 1991, which is slightly lower than the one of 2.83 [1.97-3.69] observed in RENAGO. As no change was observed in RENAGO between 1990 and 1991 , we used this figure of $2 \cdot 27$ to compute incidence by sex in 1990 .

A sex-ratio of around 4 has been observed in RENAGO since 1989. It can thus be inferred that $(2.27$ isolates $/$ lab $\times 4 / 5 \times 4193$ laboratories $=7615)+1762$ cases from STD clinics $=9377$ male cases were diagnosed in 1990 in all the French microbiological laboratories.

The true incidence of male gonococcal infections is certainly greater, as all the patients seen in private practice do not undergo laboratory investigation. For example, the frequency of laboratory testing for male patients with urethritis attending GPs was about $55 \%$ in a specific study conducted among the SGPs in the FCDN in 1991, $69 \%$ in the Aquitaine network in 1988-1990, ${ }^{13}$ $69 \%$ in the Six Department - study in $1986 .{ }^{11}$ This poses the question of how many cases of male gonorrhoea are overlooked when only microbiologically ascertained cases are taken into account. In the Six Department-study, $70 \%$ of male gonorrhoea cases were microbiologically confirmed. If we use this figure, we can consider that $7615 / 0.70=10879$ male gonorrhoea cases occurred in France in 1990, outside STD clinics. The 1762 male infections diagnosed in the French STD clinics in 1990 have to be added, giving a total number of 12641 male gonorrhoea cases in 1990, that is an incidence rate of $74.4 / 100000$ inhabitants (15-59 years).

For women, following the same process as above, 1903 female gonorrhoea cases are estimated to have been microbiologically ascertained in 1990 in the French laboratories. The 510 female infections diagnosed in STD clinics have to be added, giving a total number of 2413 cases ie an incidence rate of $14 \cdot 2 / 100000$ inhabitants (15-59 years). We have no other source of data to assess how many cases of female gonorrhoea are overlooked when only microbiologically ascertained cases are taken into account.

\section{Discussion}

French STD surveillance is based on data from different systems. These systems use different definitions of STD cases, either clinical or microbiological. They probably also involve different types of patients, as suggested by the comparison between networks of age and sex-ratio of the cases. A higher 
$\mathrm{M} / \mathrm{F}$ sex-ratio in gonorrhoea cases was observed in 1990 in Paris STD clinics (9.7) than in RENAGO in the Paris area $(6 \cdot 1)$. This is unlikely to reflect differences in diagnostic protocols between the sexes according to the health care facility. As almost all the men with urethritis and women with cervicovaginitis in Paris STD clinics undergo a microbiological investigation, this would lead us to consider the high sex-ratio of almost 10 observed in STD clinics as the reference. It appears more likely that the attendance patterns of homosexual men could explain this difference in sex-ratio between networks: $40 \%$ of male gonorrhoea cases in Paris STD clinics were homo/bisexual and a higher proportion of homo/bisexual was found among male cases in Paris STD clinics (25\%) than among male urethritis in the FCDN within the Paris area (16\%). The National Sexual Behaviour Study, which has been recently held among 20000 French residents, ${ }^{14}$ will give us useful insights to understand the attendance patterns of different groups of the population at different types of health care facilities. Moreover, the routine specification of sexual orientation of cases, whilst delicate to request from private laboratories, could easily be implemented in STD clinics.

Nevertheless, the consistency of the declining trends in gonorrhoea and male acute urethritis observed from the different networks throughout the last years reduces the possibility of a bias due to any change in attendance, notification or prescription. The number of microbiologically diagnosed gonorrhoea cases had declined both in the STD clinics and the RENAGO laboratories. This decline cannot be attributed to a change in the attendance at STD clinics or in the testing practices of the practitioners, as a decrease in the number of cases of male acute urethritis has also been reported in the GP network. This last decline would not seem to be attributable to a change in notification by the SGPs, as the number of cases of other diseases such as presumed viral hepatitis did not show the same decreasing pattern. ${ }^{8}$ The hypothesis of a rise in the number of patients with gonorrhoea who would not have access to medical care is very unlikely, given the nature of the French health system.

As in many other countries where surveillance dates back to the $1980 \mathrm{~s}$, the causes of the observed trends remain hypothetical. Paris STD clinics provide the only available data during the period preceding implementation of the Aids prevention programmes. They show a raise in some acute STDs between 1979 and 1982, which could be compatible with an increased incidence in STDs among homo/bisexual men in Paris during that period, as STD clinics appear to have been favoured by them. Without knowing with certainty the pattern of trends before 1985 in the other facilities, it is difficult to impute the whole decrease observed afterwards to the AIDS prevention programmes. Nevertheless, an increase in some acute conditions would now signify a major failure in
AIDS prevention. This could be the case in France concerning homo/bisexual men, as already reported elsewhere. ${ }^{15-18} \mathrm{~A}$ slight raise (although not significant) was observed in the proportion of homo/bisexuals among men attending SGPs with urethritis. Although the number of cases is low, rectal gonorrhoea cases in RENAGO have been more frequently reported since 1988 . They were diagnosed in different geographical areas, which excludes a change attributable to an increased detection rate by a single physician or laboratory. Men with gonococcal proctitis seemed older than men with gonococcal urethritis in RENAGO in 1990, but in Paris STD clinics during the same year, they were younger than those with gonococcal urethritis. It is thus difficult to establish from the above data whether young homosexuals are affected by this increased incidence or whether a relapse has occurred among older ones. This gives us great concern about the trend in HIV incidence among homo/bisexual men in France. Several HIV serosurveillance surveys have shown that HIV infections are still occurring among homo/bisexual men. ${ }^{19-20}$ An increasing incidence rate in male acute urethritis among homosexual men has been observed in a French cohort of HIV positive adults (SEROCO) from 1988 to $1992 .{ }^{21}$ All these data emphasise again the need for enhancing specific prevention towards homo/bisexual men.

The French STD surveillance has still some limitations. Trends in chlamydial and viral infections are available only from STD clinics and are thus still difficult to generalise. Trends in Chlamydia trachomatis should be interpreted with caution without knowing the trends in screening policies in each clinic. Numbers in STD clinics represent minimal estimates. They at least indicate that chlamydiasis is now the most frequently diagnosed STD. In a 1989 study in a family planning clinic, ${ }^{22}$ around $5 \%$ of women harboured chlamydia in cervical smears (defined as a simultaneous positivity to IF and ELISA tests). In a study among private gynaecologists in the Paris area in 1991, 2.4\% of women were found positive by using a Clearview test. ${ }^{23}$ Replication of these prevalence studies will give useful insights into the epidemiology of this infection. Moreover, a laboratory network RENACHLA ${ }^{24}$ was set up at the end of 1988 on the same model as RENAGO, in order to monitor Chlamydia trachomatis infections. It will soon provide information about recent trends in chlamydial infections diagnosed in laboratories. No increase in the number of cases of herpes simplex and genital warts has been observed since 1988 in French STD clinics. STD clinics are going to be asked to identify in their annual report first attacks of these two conditions.

Finally, incidence rates are not easy to estimate with routine data from the systems, since it was not their objective when first implemented. Major efforts have been rather aimed at maintaining a high rate of participa- 
tion among the same sample of volunteers in order to present valid trends. In this paper gonorrhoea incidence in France in 1990 has been estimated by using several sources of data. Our estimation of the gonorrhoea incidence rate was of around 74 for male and 14 for female cases/100 000 inhabitants (15-59 years). In England and Wales, where the age distribution of the population is similar, gonorrhoea incidence rates for male and female in 1990 were respectively around 71 and 47 cases $/ 100000$ inhabitants (15-59 years) ( ${ }^{16}$ and $M$ Catchpole personal data). Our estimation of male gonorrhoea incidence is close to the rate observed in England and Wales. On the other hand, the estimation for female cases, which concerns only microbiologically ascertained cases, is one third in France of that in England and Wales. Contact tracing policies differ between the two countries. In France, presumptive treatment (without a laboratory confirmation) occurs in private practice and is certainly more frequently applied to female partners of infected cases, often asymptomatic, than to male ones. Although treated, these women would have no microbiological diagnosis and would thus not be included in our sentinel networks. We may also have missed high-risk women, such as occasional prostitutes who can practice self-medication, ${ }^{25}$ but their number is unlikely to explain all the discrepancy. Finally, differences in sexual lifestyles could explain different incidence rates in gonorrhoea. The comparison of sexual behaviours from the National Studies which were held in France ${ }^{14}$ and in England and Wales ${ }^{26}$ will certainly provide useful information. Whatever the explanation, this confirms that the French STD surveillance system, although appearing quite satisfactory for trends in male infections, should be improved concerning female STDs, both in terms of selected health care services and of monitored diagnoses. Information should also be collected in some facilities to more precisely describe characteristics of people with an STD.

We are grateful to all the voluntary General Practitioners, voluntary laboratories and the STD clinics who participated in the data collection in the different systems, to $C$ Black and D Black for reviewing the manuscript and to B Hubert for useful advice.

1 Anonymous. Surveillance du Sida en France. Bull Epidem Hebdom. 1993;31:139-45.
2 Torgal-Garcia J, Martin-Bouyer G, Durrande JB. Les maladies à transmission sexuelle dans un départmen français en 1978. Bull OMS. 1981;59:567-73.

3 Renton A, Whitaker L. Using STD occurrence to monitor AIDS prevention, in Assessing AIDS prevention. EC Con preverion. in Assesing AIDS pr. HIV prevenConcerted Action in Assessing AIDS prevention. EC tive strategies. Lausanne, Institut universitaire do IUMSP, $n^{\circ} 77$.

4 Meyer L. Comment améliorer la surveillance des MST en France? Bull Epidem Hebdom. 1990;11:45-6.

5 Marshall B, Lepoutre A. Dispensaires anti-vénériens: 1985-1990. Bull Epidem Hebdom. 1992;9:39-41.

6 Meyer L, Cavelier A, Bernhardt M, et al. HIV-1 seroprevalence in patients presenting with sexually transmitted disease: results from 6 Parisian STD clinics. AIDS. 1991;5: 1148-9.

7 Valleron AJ, Bouvet E, Garnerin P, et al. A computer network for the surveillance of communicable diseases: the French experiment. Am f Public Health. 1986;76: 1289-92.

8 Massari V, Valleron AJ. Recent reduction in male urethritis in France. Am $\mathcal{F}$ Public Health. 1989;79:655.

9 Garnerin P, Valleron AJ. The French communicable diseases computer network: a technical view. Comput Biol Med. 1992;22:189-200.

10 Goulet V, Mamet JP, Rebière I, Sednaoui P, Catalan F. Le gonocoque en France en 1990. Bull Epidem Hebdom. 1992;17:73-74

11 Meyer L, Job-Spira N, Bouyer J, Bouvet E, Spira A. Prevention of STDs: A randomized trial. $\mathcal{F}$ Epidemiol Comm Health. 1991;45:152-58.

12 Maurice $S$, Salamon R, Dabis F. Telematics and sentinel health information system with general practitioners in health information system with general

13 Bulletin du réseau de médecins sentinelles. Expérience régionale Aquitaine. 1991; n48. Imprimerie Université Bordeaux 2.

$14 \mathrm{ACSF}$ investigators. AIDS and sexual behaviour in France. Nature. 1992;360:407-9.

15 van den Hoek JAR, van Griensven GJP, Coutinho RA. Increase in unsafe homosexual behavior. Lancet. 1990; 336:179-80.

16 Catchpole MA. Sexually transmitted diseases in England and Wales: 1981-1990. CDR Review. 1992;2:R1-R7.

17 Forsyth JRL, Sherrard J, Traynor P. Resurgent gonorrhoea in homosexual men. Lancet. 1990;336:878.

18 Handsfield $\mathrm{HH}$, Schwebke J. Trends in sexually transmitted diseases in homosexually active men in King County, Washing

19 Meyer L, Couturier E, Brossard Y et le groupe Prévadav. Prévalence de l'infection VIH chez les patients consultant pour suspicion de MST: PREVADAV. Bull Epid Hebdom. 1992;9:37-39.

20 Ancelle-Park R, Salamina G, de Vincenzi I, Poinsard R, Cavelier A, Brunet JB. Prévalence et incidence de l'infection à VIH chez les consultants de deux centres de dépistage anonyme et gratuit parisiens. Bull Epidem Hebdom. 1991;29:120-121.

21 Creusvaux H, Meyer L and the SEROCO Study-group. Increasing urethritis incidence in a French cohort of Increasing urethritis incidence in a French cohort of Publique.

22 Meyer L, Soulat C, Weber P, Gros I, Brousselle C. Vaginal douching and ectopic pregnancy. $\mathscr{F} A M A$. 1991;265:2670.

23 Warszawski J, Weber P, Meyer L. Clearview Chlamydia test for detection of chlamydiae in cervical specimens. f Clin Microbiol. 1992;30;2216.

24 Goulet V, Blaisot B, Mackie M, Coste E, Orfila J, Catalan F. Etude des chlamydioses en 1989 à partir d'un réseau de laboratoires d'analyses médicales. Bull Epidem Hebdom. 1990;18:115-6.

25 de Vincenzi I, Braggiotti L, El-Amri M, Ancelle-Park R, Brunet JB. Infection par le VIH dans une population de Brunet JB. Infection par le VIH dans une population de
prostituées à Paris. Bull Epidem Hebdom. 1992;47: 223-4.

26 Johnson AM, Wadsworsth J, Wellings $\mathrm{K}$, Bradshaw $\mathrm{S}$, Field J. Sexual lifestyles and HIV risk. Nature. 1992; 360:410-2. 